\title{
Constraint Condition Study of Automobile Front Side Glass Vibration
}

\author{
Yinzhi He ${ }^{a}$, Yue Lv ${ }^{b}$, Zihao Shi ${ }^{c}$, Zhigang Yang ${ }^{d}$ \\ Shanghai Automotive Wind Tunnel Center, Tongji University, Shanghai 201804, China \\ aheyinzhi@tongji.edu.cn, 'lvyuetj@163.com, 'szh3483@163.com, dzhigangyang@tongji.edu.cn
}

\begin{abstract}
Keywords: Equivalent principle, Front side window glass, Matlab-Abaqus co-simulation
Abstract. In order to study the vibration response of automobile front side window glass at high speed, its constraint condition must be determined at first. However, it is difficult to establish the boundary constraint model of the window system due to the complicated mechanical properties and the irregular shape of the side window glass seal. In this paper, a front left side window of a sedan was taken as a model. An equivalent discrete model of side window glass seal was established based on equivalent principle, by which the window seal is equivalent to series of spring constraint. Then the Matlab-Abaqus co-simulation optimization platform was set up and Python language scripts was used to make secondary development of Abaqus software. The natural frequencies of the side window glass obtained by the modal test are taken as the objective function. By fitting the stiffness of the springs, the most suitable constraint condition of the side window glass for the actual situation was obtained. Through comparing the results with simulation and experiment, the first four natural frequencies and mode shapes of the side glass are in good agreement, which verifies the effectiveness of the glass seal system discretization model by equivalent springs.
\end{abstract}

\section{Introduction}

Automobile window sealing system includes windows, seal, lift and other components. And the function of the sealing strip is to prevent the glass from falling off, to eliminate the gap between the glass and the body structure, and to improve the sealing performance of the vehicle body and noise reduction. However, the super-elastic rubber materials makes the constraint has nonlinearity characterizes in material, geometry and boundary condition. So it is very difficult to establish the boundary constraint model of the window system. Recently, scholars of domestic and overseas have done much relevant research on sealing system design and simulation analysis. Andin T [1] used spring elements instead of rubber seal to study the effect of body structure vibrations on the interior noise distribution, based on an equivalent replacement method. E.Dikmen [2] built a super elastic material model of a door seal to predict the dynamic characteristics of a door. Park J [3] analyzed the influence of mechanical properties of sealing strips on noise and vibration characteristics of a side window glass under turbulent flow excitation. Wenfeng Zhu [4] made the glass run equivalent to elastic constraint, and used compression load deflection tests of unilateral lips to determine the spring stiffness.

In this paper, based on equivalent principle [5], an equivalent discrete model of a front side window glass seal of a sedan was established, by which the window glass seal is considered equivalent to series of spring constraint. Meanwhile the spring stiffness is quickly fitted out by Matlab-Abaqus co-simulation and intelligent algorithm, which can be acted as the basis for further research on the vibration response of the front window glass under high speed wind excitation.

\section{Modal Test Analysis}

In order to obtain the dynamic characteristics of the front window glass system and provide the objective for stiffness fitting of discrete models, a modal test of the side window glass under constrained baseline state was carried out with multi-point excitation and single point response. In theory, multiple excitation points can be more accurate to stimulate the structure of the various modes. 
However, the increase of the excitation points will cause the increase of the calculation time. In this case, 34 excitation points were arranged on the surface of the front side window glass averagely. A response point was set at point 31 in the upper right corner of the window. During the test, an accelerometer was pasted at point 31 to collect the acceleration response signal of the side window glass under the excitation of an impulse force from a hammer hit. Test equipment and test point's location shown in Figure 1.

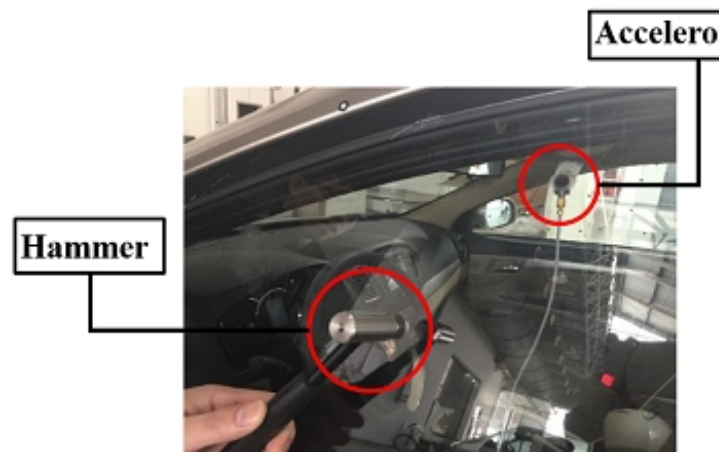

a) Test equipment

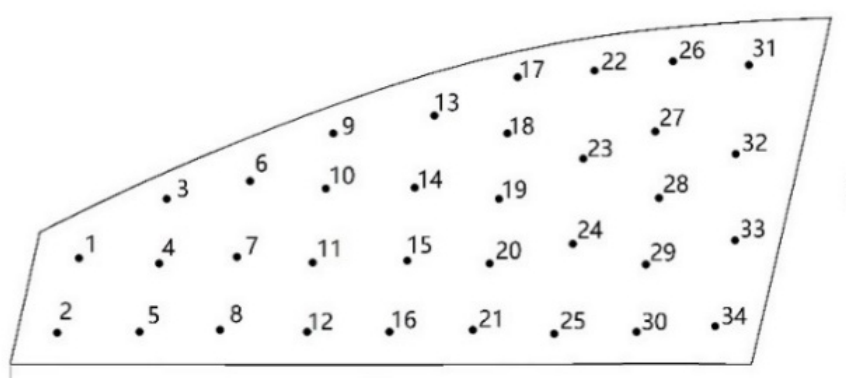

b) Location of test points

Fig.1. Test equipment and test points location

ATP08 system was used to acquire test data, and the sampling time is $5 \mathrm{~s}$, the sampling frequency is $24 \mathrm{KHz}$. Based on the system requirements, the model of side window glass was established according to the test points coordinates at first. Then so called hammer hit method was used to collect transfer functions of all the excitation points to the response point. With curve-fitting procedure, the series of natural frequencies, damping ratio and mode shapes were acquired. Table 1 shows the four nature frequencies and damping ratio, the corresponding first four mode shapes are shown in Figure 2.
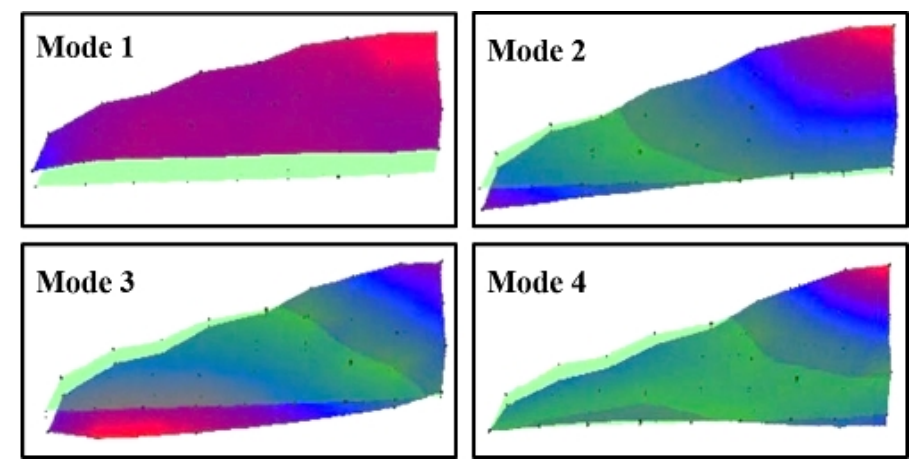

Fig.2. First four mode shapes

Table 1. Natural frequencies and damping ration of test results

\begin{tabular}{|c|c|c|}
\hline $\begin{array}{c}\text { Modal } \\
\text { number }\end{array}$ & Natural frequency $(\mathrm{Hz})$ & Damping ratio (\%) \\
\hline 1 & 40.8 & 4.48 \\
\hline 2 & 64.9 & 3.66 \\
\hline 3 & 80.9 & 6.14 \\
\hline 4 & 106 & 5.57 \\
\hline
\end{tabular}

\section{Discrete Modeling of Window Sealing System}

Since the seal constraint has nonlinearity characterizes in material, geometry and boundary condition, it is very difficult to establish directly the boundary constraint model of the window system. So in this 
paper, based on equivalent principle, the window glass seal is considered equivalent to series of spring constraint.

Boundary Constraint Condition Equivalence Principle. The side window glass seal acts as a good supporting, sound insulation and sealing through extrusion with the window. Since the seals are mostly made of EPDM rubber super elastic rubber material, it makes the material has nonlinear property; due to great deformation of the sealing strip in the working process, the relationship between force and elongation does not accord with the traditional Hooke's law, hence the sealing strip has the typical geometric nonlinearity; the contact and friction between the seal and the window glass are non-linear with boundary conditions $[6,7]$ as well.

The non-linearity of the sealing strip makes it difficult to establish the boundary constraint model of the window system. Therefore, based on the equivalent principle, glass run and weather strip seals are discretized into series of spring constraint. The spring stiffness can be obtained with fitting procedure by the Matlab-Abaqus simulation platform, which based on genetic algorithm.

Establishment of Finite Element Model of Side Window Glass. To fit the spring stiffness, it is necessary to establish the finite element model of the front window glass at first. Its complete geometry is shown in Figure 3. The model is meshed with triangular shell elements, and the minimum mesh size is $5 \mathrm{~mm}$. The properties of the side window glass are shown in Table 2.

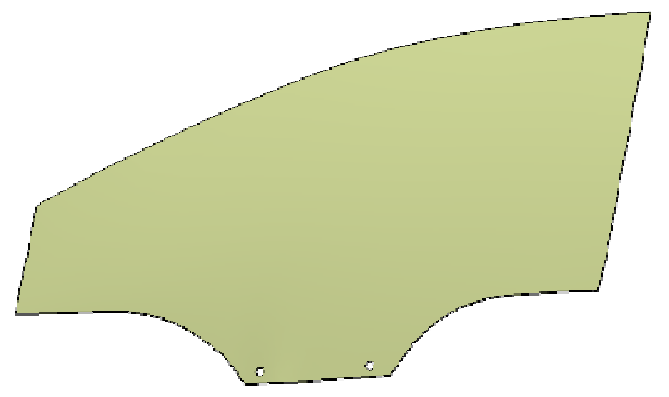

Fig.3. Side window glass model

Table 2. Material property of side window glass

\begin{tabular}{|c|c|c|c|}
\hline Material & Density $\left(\mathrm{kg} / \mathrm{m}^{3}\right)$ & Elastic modulus $(\mathrm{GPa})$ & Poisson ratio \\
\hline Toughened glass & 2260 & 72 & 0.20 \\
\hline
\end{tabular}

Spring units are arranged at each mesh node of the seal strip around the window, one end of the spring is connected with the mesh node, the other end is fixed. The direction of spring compression is normal to the side window. Since the position of the test point is close to that of the spring element, the displacement at the test point can be approximately expressed as the compression of the spring element which is near the test point. Because the first mode of the modal test is similar to the rigid displacement of side window glass, the displacement (that is, the spring unit compression) of test point which around the glass can be obtained by the first mode. Furthermore, based on the inverse proportional relationship of spring stiffness and compression, the spring stiffness which is near the test point 2 can be set as the reference stiffness $\mathrm{K}_{1}$, and the rest of the stiffness are shown in Table 3 . 
Table 3. Spring stiffness where near the test points

\begin{tabular}{|c|c|c|c|}
\hline Test point & Stiffness & Test point & Stiffness \\
\hline 1 & $0.819 \mathrm{~K}_{1}$ & 17 & $0.598 \mathrm{~K}_{1}$ \\
\hline 2 & $\mathrm{~K}_{1}$ & 21 & $0.630 \mathrm{~K}_{1}$ \\
\hline 3 & $0.709 \mathrm{~K}_{1}$ & 22 & $0.580 \mathrm{~K}_{1}$ \\
\hline 5 & $0.685 \mathrm{~K}_{1}$ & 25 & $0.644 \mathrm{~K}_{1}$ \\
\hline 6 & $0.678 \mathrm{~K}_{1}$ & 26 & $0.543 \mathrm{~K}_{1}$ \\
\hline 8 & $0.637 \mathrm{~K}_{1}$ & 30 & $0.676 \mathrm{~K}_{1}$ \\
\hline 9 & $0.658 \mathrm{~K}_{1}$ & 31 & $0.521 \mathrm{~K}_{1}$ \\
\hline 12 & $0.655 \mathrm{~K}_{1}$ & 32 & $0.558 \mathrm{~K}_{1}$ \\
\hline 13 & $0.648 \mathrm{~K}_{1}$ & 33 & $0.641 \mathrm{~K}_{1}$ \\
\hline 16 & $0.636 \mathrm{~K}_{1}$ & 34 & $0.677 \mathrm{~K}_{1}$ \\
\hline
\end{tabular}

As the real vehicle window glass and the door is connected by pin, so the pin hole constraint of the glass bottom is also set equivalent to spring constraints. And the stiffness is set to $\mathrm{K}_{2}$, as shown in Figure 4.

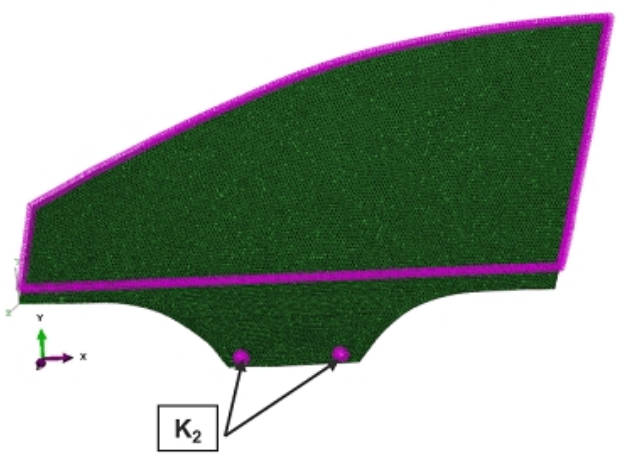

Fig.4. Spring stiffness of pin hole

\section{Equivalent Spring Stiffness Fitting}

In order to simulate the most suitable spring stiffness of side window glass constraint, and to reduce the cumbersome and error probability of data processing with high research and analysis efficiency, Matlab-Abaqus co-simulation platform is used to find the optimal solution of spring stiffness, based on genetic algorithm.

Control Target. In order to simulate the real side window boundary constraint and obtain the optimal spring stiffness, the standard deviation of the natural frequencies is taken as the control target, since natural frequency is the inherent characteristic of system. The definition is shown in equation (1). It can be seen from the equation, the closer the standard deviation is to zero, the better are the optimization results.

$$
S=\sqrt{\frac{\sum_{i=1}^{n}\left(X_{i}-X_{i}^{\prime}\right)^{2}}{n}} .
$$

Where $S$ is the standard deviation, $X_{i}$ is the simulation value of the $i^{\text {th }}$ order of natural frequency, $X_{i}^{\prime}$ is the test value of the $i^{\text {th }}$ order of natural frequency, $n$ is the number of samples, here the first 4 order of nature frequencies are taken into consideration.

Establishment of Matlab-Abaqus Co-simulation Platform. The main idea of using Matlab-Abaqus co-simulation platform for side-window glass spring stiffness fitting is using Matlab 
to modify Abaqus finite element model (INP file), and calling Abaqus/CAE for calculation. The processing results will be returned to Matlab after Abaqus calculation is completed, then Matlab genetic algorithm toolbox is used to do iterative calculations. Finally the best spring stiffness [8,9] can be fitted. The flowchart of co-simulation method is shown in Figure 5.

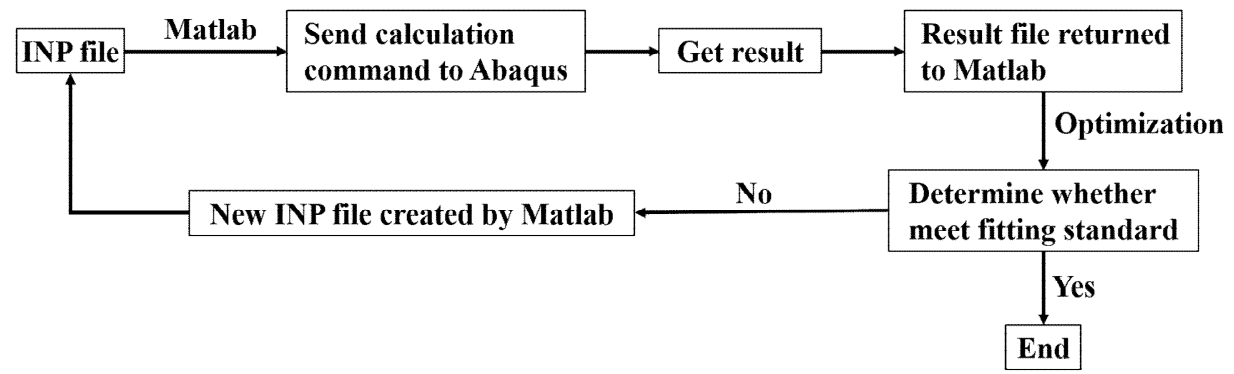

Fig.5. Matlab-Abaqus co-simulation method

Since Matlab cannot recognize the calculation results (ODB file) from Abaqus in the process of fitting, it cannot directly extract Abaqus ODB file for standard deviation calculation. Abaqus provides a Python script interface, and Python script has the characters of scalability, portability and embed ability, so in this paper, Python language scripts is used to make secondary development of Abaqus software [10]. Finally Matlab reads Abaqus calculation result through the Python script, and the data extraction is finished.

Simulation Results Analysis. The spring stiffness obtained by the above mentioned fitting method is as follows: $\mathrm{K}_{1}=0.734 \mathrm{~N} / \mathrm{mm}, \mathrm{K}_{2}=41.25 \mathrm{~N} / \mathrm{mm}$. The control target (natural frequency standard deviation) converges to 4.0, and the results are shown in Figure 6. The standard deviation reaches a steady value after 13 iterations. And the first four natural frequencies of the final fitting model are shown in Table 4.

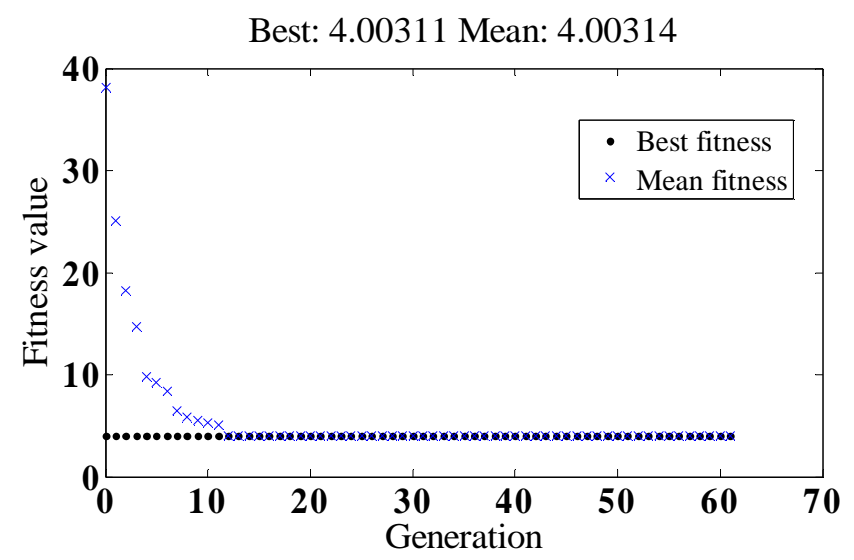

Fig.6. Fitness result of natural frequency standard deviation

Table 4. The first four natural frequencies of the final fitting model

\begin{tabular}{|c|c|c|}
\hline Modal number & Natural frequency- test $(\mathrm{Hz})$ & Natural frequency- simulation $(\mathrm{Hz})$ \\
\hline 1 & 40.8 & 47.2 \\
\hline 2 & 64.9 & 64.4 \\
\hline 3 & 80.9 & 79.2 \\
\hline 4 & 106 & 101.3 \\
\hline
\end{tabular}

\section{Results Verification}

In order to verify the rationality of the side window glass equivalent constraint method, comparison of the results between experiment and simulation with finite element method is done. Figure 7 shows the 
comparison of the first mode shapes, it is clear that the side window glass with discrete equivalent seal and the actual seal has a good consistency in every vibration mode. Therefore, it is effective and reasonable to replace the actual seal with the equivalent spring, and fit the spring stiffness from Matlab-Abaqus co-simulation platform.
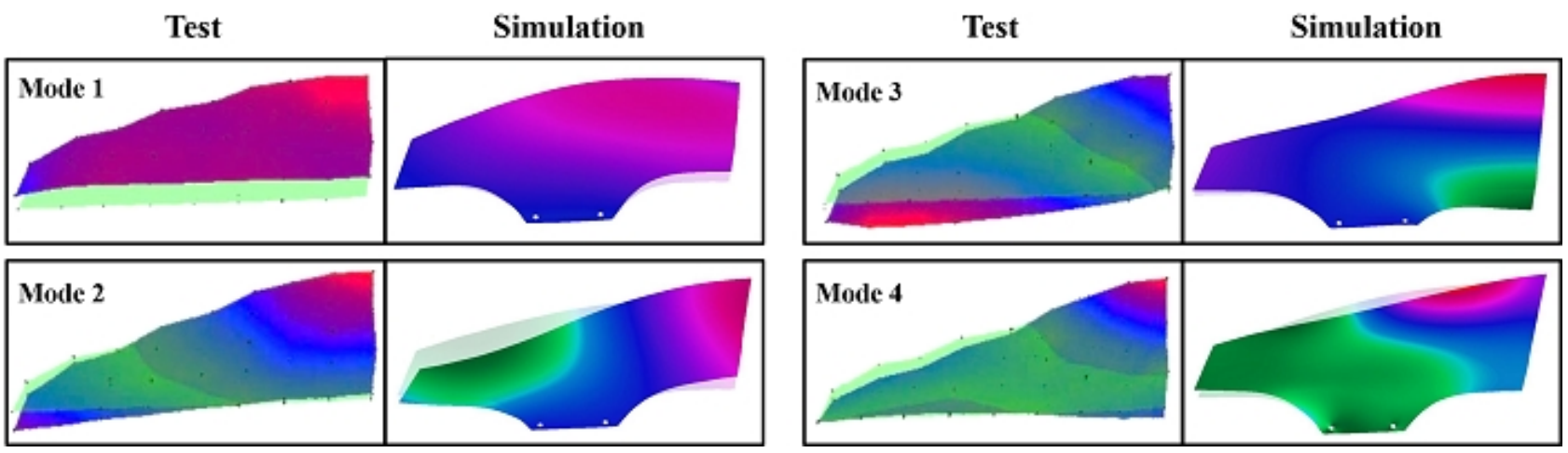

Fig.7. First four mode shapes for test and simulation

\section{Conclusions}

1) Due to the complicated mechanical properties and the irregular shape of the side window glass seal, the sealing strip is set equivalent to series of spring constraint based on equivalent principle, so that establishment of the discretization model of the window sealing system is realized.

2) The spring stiffness can be more accurately fitted on the Matlab-Abaqus co-simulation platform based on genetic algorithm. By comparison of the simulation and test results, it is demonstrated that the first four natural frequencies and mode shapes of the two methods are in good agreement. Hence the rationality of discretization modeling of window sealing system through equivalent spring is validated.

\section{Acknowledgements}

This work was financially supported by the National Natural Science Foundation of China (51575394).

\section{References}

[1] Tuncer A, Anlas G, Yilmaz Y: Journal of Vibration and Acoustics, 2014, 136(4): 041018.

[2] Dikmen E, Basdogan I: Vehicle System Dynamics, 2008, 46(11): 975-990.

[3] Park J, Mongeau L, Siegmund T. Effects of window seal mechanical properties on vehicle interior noise[R]. SAE Technical Paper, 2003.

[4] Wenfeng Zhu, Peijiang Lin, Hui Zhou, et al: Journal of Mechanical Engineering, 2015, 51(20): 171-176. In Chinese.

[5] Xiuchang Huang, Shiyin Xu, Hongxing Hua, et al: Journal of Shanghai Jiaotong University, 2013 (2): 173-179. In Chinese.

[6] Olsson A K. Finite element procedures in modelling the dynamic properties of rubber [M]. Lund University, 2007.

[7] Xiaoyong Pan. An Investigation on Calculation and Modeling Methods for Dynamic Properties of a Rubber Isolator [D]. Zhejiang: Zhejiang University of Technology, 2009. In Chinese. 
[8] Systèmes D: Simulia Corp. Providence, RI, USA, 2007.

[9] Kurma S. Vibration and stability of composite panels with geometrical discontinuities [D]. , 2013.

[10]Jinfeng Cao, Xuchun Wang, Liang Kong. The Application of Python Language in Abaqus [M]. Beijing: China Machine Press, 2011. In Chinese. 